\title{
Population Toxicology - an Example of Super-individual Approach in Human Studies
}

\author{
Csaba Varga ${ }^{1, *}$, Bettina Piko² \\ ${ }^{1}$ Department of Environmental Health, Institute of Public Health Medicine, Faculty of Medicine, University of Pécs, Pécs, 7624, Hungary \\ ${ }^{2}$ Department of Behavioral Sciences, Faculty of General Medicine, University of Szeged, Szeged 6722, Hungary
}

\begin{abstract}
Authors prove the need of super-individual approach in environmental toxicity studies since the specific human-environment interactions can only be interpreted using basic rules of ecology. The main goal of the paper is to collect, systematize and weigh several new factors of vulnerability of populations to the environmental exposures. Human populations influence and are influenced by the physical settings and cultural systems in which they live. That is; social, cultural, historical, economic etc. determinants are at least so important confounders as genetic or other kind of vulnerability of the population. Authors demonstrate different outcomes of environmental exposures depending on mainly non-material factors. Good examples, when social factors may lead to cultural maladaptation that contributes to the appearance of cognitive or other dysfunctions through biological mechanisms (fiber or metal toxicity) in case of children's elevated blood lead or disproportional exposure of subpopulations to asbestos. The novel super-individual approach of human environmental toxicology proposed here - as an integrated part of both preventive medicine and ecology - based upon the multiple nature of humans, and will join the present change of paradigm in human environmental studies.
\end{abstract}

Keywords Vulnerable Populations, Environmental Exposure, Ecotoxicology, Environmental Toxicology, Environmental Health, Inequities, Socioeconomic Status, Minority Health

\section{Introductory Remarks}

Working as a researcher and university teacher on the frontiers of environmental, social and health sciences one has often faced the following questions: Do medical students or the future physicians need any super-individual approach and attitude? They will treat and cure individuals, won't they? It is the time to revise these or similar views. Today's medicine does not merely mean curing at all, despite the public believes it[1]. Medical science has three main branches, and the curative (or rather clinical involving diagnostics) medicine is only one of them. Prevention (preventive medicine) is equally or - at least - not less important field, involving disciplines to be concerned at the super-individual level, e.g., epidemiology of infectious and non-infectious diseases; medical demography, geography and sociology; social medicine; behavioral science; etc. (Finally, the third and youngest branch is the regenerative medicine, indicated by the stem-cell research and treatments.)

One part of disorders is of environmental origin, another part is basically genetic, but nowadays we should rather speak about interactions of genetics and environment. Environmental health and epidemiology focus on these inter

* Corresponding author:

chemsafety@freemail.hu (Csaba Varga)

Published online at http://journal.sapub.org/env

Copyright (C) 2012 Scientific \& Academic Publishing. All Rights Reserved actions. Their research methodology considers populations (communities) as subjects of the studies, although the exact definition of the term "human population" is highly debated. (Nota bene: medicine rarely applies biological principle of population, using preferably this term for a simple set or abundance.)

The policy makers have learned well the elemental truth up to now: prevention is much more cost-effective than medical treatment. But let the approach be political, economic or scientific, the knowledge of methods, theory and practice of prevention is essential for the students and medical doctors, as well.

\section{Health and Medicine: an Ecological Viewpoint}

The modern concept of health and disease is a basic principle of preventive medicine. According to the earlier definition of the World Health Organization (1946) "Health is a state of complete physical, mental and social well-being and not merely the absence of disease or infirmity."[2] This definition calls attention to the fact that being healthy involves much more than simply determining illness as it also encompasses well-being. Another advantage of it lies in the fact that it emphasizes complexity and dimensionality of health. All these aspects of the definition proved to be useful for both clinical medicine (e.g., in assessments of quality of 
life) and preventive medicine (e.g., in mapping biopsychosocial mechanisms of risk factors). However, this classical definition has been heavily criticized since the WHO introduced it. One of the main criticisms is that it refers to well-being, which corresponds much more closely to happiness than to health.

Therefore, to remove the conceptual difficulties and take the criticisms into account, the WHO (1984) completed the original definition with some new aspects: "Health is the extent to which an individual or a group is able to realize aspirations and satisfy needs and change or cope with the environment."'[3] Based on these new aspects, health may be seen as a resource for everyday life involving both personal and social resources as well as physical capacities. This model suggests an ecological perspective, i.e. health is a state of equilibrium between the individual and the physical, biological and social environment, compatible with full functional activity. Health in this case corresponds more to harmony with environment.

The modern ecological definition of health brings about changes in our viewpoints related to disease-inducing mechanisms and preventive methods as well. From ecological point of view, adaptation has become a key element in health maintenance[4]. Adaptation is the process of accommodation that takes place between an organism and its environment both physical and social/cultural. However, while the biological adaptation may be well measurable by reproductive success since adaptive traits ensure the survival of the individuals, the cultural/social adaptation is far less clear and simple to measure. What are the characteristics of a well-adapted population? Is it a successful adaptation when a population is successfully able to cope with the stresses of the environment but its subsistence activities do not threaten immediate degradation of the environment?

In modern ecology, we should take into account that human populations influence and are influenced by the physical settings and cultural systems in which they live. Thus, exposure to harmful disease-inducing agents requires a super-individual approach emphasizing the interplay between the natural and cultural environment. Exposure to toxic agents in modern urban settlements may be an impressive example of this super-individual approach.

\section{Environmental vs. Ecotoxicological Aspects}

Environmental toxicology, a field of environmental health - in traditional sense - investigates physiological alterations of the individual upon impacts of physical, chemical and biological contamination of environment. Based on this statement one can simply classify it as a field of physiology (or pathophysiology). It may not be anything else than descriptive: human experiments cannot be performed.

On the contrary, ecotoxicology is $a b$ ovo experimental: a set of ecotoxicity tests are carried out in laboratories of regulatory authorities all over the world, investigating polluted environmental samples or notifiable new chemicals. But the basic difference is hidden somewhere else: ecotoxicology examines effects on populations of microorganisms, plants and animals, or higher super-individual organization levels. In strict sense, even the Salmonella Ames mutagenicity test, "the stethoscope of the $21^{\text {th }}$ century" for genetic toxicology and prevention[5], is also an ecotoxicity test.

\subsection{Asbestos Exposure: an Example}

During human toxicity studies the question has often arisen on the possibility of modeling exposure of populations. The environmental asbestos exposure may be an éclat example. The extensive mining and application of this fibrous mineral have caused world-wide pollution since the middle of the 20th century. The inhaled asbestos fibers are known as carcinogens, but the scenario of a massive inhalation can only take place under occupational conditions. In the inhalation and instillation studies animals are treated with standard UICC asbestos fibers almost exclusively. These are very short fibers having homogenous distribution, and were produced by grinding of existing (mined) asbestos specimens. These samples in laboratory studies are primarily used to generate comparable results. But, what is the realworld exposure situation? The whole population is rather involved via ingestion of fibers, as a consequence of environmental (non-occupational) exposure: e.g. contaminated drinking water. The size-distribution of these fibers is completely differs from the UICC samples. The "commercial-size" asbestos fibers are mainly non-respirable, so the primarily inhaled fibers (from corrosion of asbestos-cement products: roof-sheets, insulations, break-linings, etc.) can also be ingested later, as a consequence of operating mucociliary escalator in the airways. Since the whole population is involved in the ingestion way of exposure, the oral exposure can be considered as the main route. This statement may not be certainly true in all cultural environments. Ingestion is conventional e.g., in the Jewish-Christian culture, but in some other societies sputum is a well-tolerated human excrete (see betel-consumption). Therefore mineral fiber research and especially the risk assessment studies should consider this approach to avoid a possible dead-end street[6].

The situation is more or less similar in the case of nanoparticles and nanotubes which mean a new great challenge for environmental health. Their size-distribution is similar to the small asbestos fibers and elementary fibrils. Research is nowadays restricted to the inhalation studies; nevertheless aggregation of particles is a well-known phenomenon. (Preparing homogenous suspensions from nanoparticles is practically impossible, and the aggregated particles and especially the nanotubes can behave as environmental asbestos fibers.) [7, 8]

Can one consider the abovementioned super-individual approach of particle-toxicology as a specific field of ecotoxicology ("human ecotoxicology")? Perhaps yes, but 
the definition could be applied with severe restrictions. Population toxicology seems to be a much more exact expression. If, however, we try to transplant "ecotoxicology" to human conditions we would inevitably face to an abundance of non-material environmental factors: social and historical determinants.

\subsection{Environmental Epidemiology Vs. Population Toxi- cology}

While environmental epidemiology can only follow the cases, population toxicology should accumulate basic information on possible toxicants and routes of exposure at population level. The super-individual approach, of course, is an important attribute of epidemiology, as well. But epidemiological studies should be based on firm, extensive and causative assumption of correlations previously suggested by empiria (observations), experiments or statistics. The final aim of all preventive measure is identification and assessment then avoidance or decreasing the risk. The analogy is clear with ecotoxicology focusing preventive measures onto the other living organisms. As human is also an integrated part of the ecosystems and biosphere, but is a societal being as well, it requires specific consideration due to its double state.

\section{The individuals and Their Environ- ment: An Ecological Model to Stress}

As the microbiologist René Dubos (1965) stated: illness is a product of maladaptation[9]. Maladaptation, on the other hand, may lead to disease. Modernization brought about significant changes in social environment that might challenge populations in the modern world through elevating stress and led to increase the threat of maladaptation. The process of adaptation may be well understood through an ecological model to stress that explains the interplay between the individuals and their environment.

The word stress has become very popular since the Hungarian-born physiologist, Hans (János) Selye wrote his book on the stress of life in 1956. In his view, stress represents the general response of the organism to environmental demands which is neither positive nor negative in nature but merely a physiological mechanism that covers an alarm reaction which prepares an individual for action and adaptation. Disease may be viewed as a possible negative consequence of the individual's or the population's response to the environmental demands, due to the inadequate adaptation to changes of the environment. Environmental processes are stress-inducing when they overwhelm adaptation capacity[10].

Stress has become a symptom of modern life since there are a number of challenges of our modern life which press human beings to permanent adaptation. This type of adaptation, however, often overwhelms their capacity. The current phase of civilization, the continuous rapid changes are per- manently create new challenges. On the other hand, the loss of traditions and the alienation of human relationships are permanently creating new conflicts among the individuals and society. As a consequence, not only more stress we should face but also it is getting more difficult to cope with stress stemming from the environment in modern society. The question is how societies can cope with the "stress epidemic" and develop social and public health strategies by promoting an environment that provide healthy conditions for the population[11].

Based on the stress model, there are societies which are vulnerable from ecological point of view[12]. Those experience rapid changes in their social structure that may lead to chronic stress are in particular vulnerable since they need to adapt from time to time. For example, Eastern Europe's new democratic societies should face enormous challenges to react to rapid changes. In addition, their nondemocratic traditions make it even more difficult for the individuals and communities to adopt successfully. The essence of successful adaptation is whether the individuals or communities have the control over a stressful situation or not[13]. If they face a situation as controllable, they are able to actively work on the problem-solving. If people find the situation frightening and uncontrollable, the feeling of anxiety will emerge and they will refuse to engage in active coping. Chronic stress and anxiety would create a feeling of inability to actively control situations perceived as threatening. Therefore, they cannot successfully adapt to environmental demands. In the background of morbidity and mortality data of the region of Eastern Europe, adaptation problem at population level is also present.

On the other hand, what societies can we call as well-adapted? According to Wilkinson \& Pickett (2009), life expectancy at birth is the highest not in countries with the highest GDP but with a more egalitarian distribution of GDP, such as Sweden or the Netherlands[14]. These societies are 'healthy' societies in terms of being well-integrated where social equity and social capital reassures a healthy functioning of social environment and helps with a better social adaptation.

We should also note here that we should make differences between unavoidable health inequalities (i.e., general differences in health status and exposure levels due to individual predispositions) and inequities (i.e., avoidable socio-economic differences in health and health-related environmental hazards, e.g., access to screening)[15]. However, more research is needed to clarify what proportion of health inequalities may be tolerated and accepted; what factors contribute to equity; and the possibility of modification of effect stemming from different risks for different socio-economic groups. The process of policy making for handling inequalities and inequities is influenced by historical roots of societies.

\subsection{Genotypes and Phenotypes}

As we see, different factors can be considered as bases of 
the adaptive power of a population. Contemporary population genetics and genomic epidemiology has revealed numerous crucial facts on genetic background of adaptation or vulnerability. The recent levels of physiological heterogeneity and genetic diversity of human populations are consequences of the restricted evolutionary burden: the lack of natural selection, indeed, a certain counter-selection caused by high quality and continuous development of medical care. Let us think of the broad variation in metabolism of xenobiotics: environmental chemicals and pharmaceutical products. This fact is well indicated by the new patient-tailored pharmacology. The novel trend of personalized medicine seeks to provide an objective basis for consideration of individual differences. (Traditionally, it has been limited to the consideration of a patient's family history, social circumstances, environment and behaviors in tailoring individual care.) It is true that genomics, proteomics and metabolomics can suggest individual strategies to avoid environmental and occupational risks but hygienic toxicology cannot simply work at personal level. Extrapolation of data collected from animal studies does not give the opportunity to set individual thresholds of exposures, but metabolic/genetic patterns of subpopulations can be taken into consideration and modeled by targeted selection of animal strains in the laboratory experiments. This way, the uncertainty widely used in hygienic and occupational toxicology can significantly be decreased calculating distinct exposure limits for the subpopulations. (This may also be reminiscent of pharmacology regarding different efficiency of some drugs in specific ethnic/race groups.) The present situation of uniform hygienic exposure limits based upon too high uncertainty calculations cannot be kept any longer, especially at the state-of-art level of science.

\subsection{Vulnerability Stemming from the Interplay Between culture and Biology}

Cultural adaptation has a meaning of how people in everyday life try to adapt to challenges coming from society. Societal norms and cultural value systems provide a guideline for behavioral decisions and coping strategies people use in their thriving for better living. Within a society there are tremendous social inequalities in health that may stem from differences in health-related exposures. These health risks, although through biochemical mechanisms, may be related to disease in connection to socio-cultural processes. Nevertheless, socially disadvantaged groups lack the appropriate social resources to cultural adaptation and therefore, may face higher level of vulnerability to a number of toxic agents. For example, as Fitzpatrick and LaGory[16] outline, there are "unhealthy places" in modern towns. The concentration of poverty and the segregation neighborhoods by social class (and ethnicity and race somewhere) is a powerful force in reshaping social inequalities in health. Poor neighborhoods in these places represent elevated levels of physical hazards and risks in the residential environment. Disadvantaged populations are disproportionately exposed to a wide range of environmental health hazards. These include, for example, air quality, noise pollution, or toxic waste dumping[17].

A good example is the health consequence of heavy metal toxicity among children in disadvantaged neighborhood, that is, "unhealthy places". For example, it is now scientific evidence that lead is an environmental contaminant; and the majority of epidemiological research on the health effects of lead has been focused on children, because they are more vulnerable to lead than adults. In children, an elevated blood lead is associated with reduced Intelligence Quotient (IQ) score[18]. This may be a source of a number of problems with cognitive ability that may generate social inequalities. Thus, this health risk is connected with the children's environmental exposure to lead as a health risk is connected to economic segregation and detrimental building. As it seems, social factors may lead to cultural maladaptation that contributes to the appearance of cognitive dysfunctions through biological mechanisms (heavy metal toxicity).

\subsection{Cognitive Vulnerability}

Not surprisingly, cognitive factors play an increasing role in modern society of information. We should also note here that social inequalities in health can not only be attributed to differences in financial resources. The Black report argued for four possible explanations of health inequalities: material circumstances, artifactual effects, behavioral-cultural factors, and social selection[19]. A number of potential pathways through which SES influences one's health have been found in research (e.g., work environments, stress, health behaviors and other psychosocial variables). Increasingly, there is some evidence that lower SES persons perceive themselves as less in control of external events, and that such beliefs are associated with poorer health[20]. It might be assumed that these measures represent important cognitive assessments of social processes. Two possible explanations for this social status - health link can be argued. First, beliefs and attitudes vary by SES strata, or second, the cognitive evaluation of people influenced by their personality that can have a profound impact on attitudes and beliefs. Research done by Kopp and associates[21] supports the importance of the psychosocial processes in understanding the relationship between SES and health. In this study they argued that the relationship between the general health status of Hungarian adults and their level of socio-economic deprivation was a function of the severity of their life dissatisfaction and depressive symptomatology. As such, people in disadvantaged neighborhoods are not able to cope with the challenges from their culture. As a consequence, due to their culturally maladaptive behavior, they become more vulnerable to biological or chemical environmental threats, for example, toxic agents. Quite often, these people are simply not aware of the health hazards and even if they are so cannot change their environment[22].

\subsection{Historical aspects of vulnerability}

The realized niche of a population characterizes well its power in the competition of coexisting ones. In biology, a 
population is a single and unique representative of a species in the particular ecosystem with its specific environmental requirements. Overlapping needs of energy and nutrient sources decrease the fundamental niche of the population to the realized one. Considering human populations social and historical aspects sophisticate the picture even further. Human populations (nations) with overlapping environmental (economic, geographic, ideological, etc.) requirements belong to the same species. Competition of Homo sapiens populations is sometimes called aggression and war (while expansion of habitat is sometime called Lebensraum,[neo]colonization, etc.)[23]. Even the stormy history of Europe in the 20th century delivers several examples for formation of second-class national minority groups in newly established countries, pressured morally, culturally; who neither have the right to healthy environment, nor the fair medical care. (Care in non-native language is a significant disadvantage indicated by well-defined health indices.) Consequently, the "historical" or "national minority" confounder should also be considered in some geographical regions[24].

\subsection{Other Factors}

Other important aspects of vulnerability, susceptibility or sensitivity as physical and physiological state (in close correlation with biotransformation capacity), effects of life stage, pre-existing diseases, etc. are entirely reviewed by Sacks et al.[25] with a special reference to particulate matter exposures.

\section{Experimental Design Based Upon the Human Super-Individual Approach}

Modeling the human situation in experimental studies has always been the final aim of hygienic toxicology. Building an effective strategy to assess the actual, population or subpopulation based risk is only possible by using the whole available armament of experimental toxicology. Nowadays the scientist can choose from a large list of selected or genetically engineered (incl. transgenic) animal strains for the studies. After a deep analysis of the exposure and all abovementioned characteristics of the exposed population(s) the experimental study can effectively be designed. If the exposed populations are of known ages, animals of the particular (animal) ages should be used. It is also true for the physical-physiological state and the sex (but not gender). These are, as a matter of fact, traditional elements of animal experiments. Investigation on health effects of exposure to populations with pre-existing diseases (cardiovascular, obesity, diabetes, etc.) can be regarded as routine procedure as well, since such strains of mice, rats, or other species are available. Theoretically, human genetic polymorphisms (sometimes also reflecting to race) could also be modeled but the differences in metabolic pathways of xenobiotics and other environmental chemicals may be significant in animals.
(Polymorphism and consequently the phenotypic alteration in metabolism is a possible main cause of vulnerability to chemical exposures.) Another obstacle is little alterations of enzymatic activities - resulting moderate variation in risks calculated - in case of human polymorphisms. These little differences may be effectively masked by the uncertainty of interspecies differences. In case of other target polymorphisms (e.g. fibrinogen genes, DNA repair, etc.) the situation is, nevertheless, more promising[26].

\section{New Challenges, New Answers}

Accelerated alteration of the global environment generates urgent need for adaptation of human societies to the chemosphere and infectosphere (emerging infections!), involving preventive measures at population (society, community) level. This super-individual attitude is not yet unfamiliar in some medical fields. For example conservation medicine is a relative of (and derived from) conservation biology. Dealing with correlations of human, animal and ecosystem health, it tries to explain the causes, nature and possible consequences of emerging diseases and global changes. Representatives of the field recognized the need to take a holistic approach to monitoring and assessment that transcends traditional scientific or medical disciplines. This is a basic tenet of conservation medicine and should lead to the identification of new patterns or connections that perhaps went unnoticed[27].

The novel super-individual approach of human toxicology proposed here - as an integrated part of preventive medicine - will join this change of paradigm. One important final aim of public and environmental health and prevention studies is calculating useful data for the regulation authorities. A "fine adjustment" of exposure limits and acceptable risks can only be based upon social: cultural, anthropological, historical, etc. research of human populations. In the new age of revolutionary "omics", the extremely analytical approach ignoring specificities of the $H$. sapiens populations can easily mislead us.

\section{REFERENCES}

[1] Piko, B. \& Stempsey, W. E. (2002). Physicians of the future: Renaissance of polymaths? J Roy Soc Promo Health, 122, 233-237.

[2] World Health Organization. 1946. Constitution. Geneva: World Health Organization.

[3] World Health Organization. 1984. Health promotion: A WHO discussion on the concept and principles. Geneva: World Health Organization.

[4] Piko, B. F. \& Kopp, M. S. (2002). Behavioral medicine in Hungary: Past, present and future. Behav Med., 28, 72-78.

[5] Claxton, L. D., Umbuzeiro, G. A. \& DeMarini, D. M. (2010). 
The Salmonella mutagenicity assay: The stethoscope of genetic toxicology for the 21st century. Environ Health Perspect., 118, 1515-1522.

[6] Varga, C. (2005). Can one assess genotoxic and carcinogenic risk of asbestos without mentioning ingested fibres? Mutation Res., 572, 173-174

[7] Szendi, K. \& Varga, C. (2008). Lack of genotoxicity of carbon nanotubes in a pilot study. Anticancer Res., 28, 349-352.

[8] Varga, C. \& Szendi, K. (2010). Carbon nanotubes induce granulomas but not mesotheliomas. In Vivo, 24, 153-156.

[9] Dubos, R. (1965). Man adapting. New Haven: Yale University Press.

[10] Selye, H. (1956). The stress of life. New York: McGraw-Hill.

[11] Duhault, J. L. (2002). Stress prevention and management: A challenge for patients and physicians. Metab., 6(S1), 46-48.

[12] Lundberg, U. (2008). Stress and (public) health. Int Encycl Publ Health 241-250.

[13] Piko, B. (2004). Interplay between self and community: A role for health psychology in Eastern Europe's public health. J Health Psychol., 9, 111-120.

[14] Wilkinson, R.G. \& Pickett, K. (1996). The spirit level: Why more equal societies almost always do better? London: Allen Lane.

[15] Martuzzi, M., Mitis, F. \& Forastiere, F. (2010). Inequalities, inequities, environmental justice in waste management and health. Eur J Publ Health, 20, 21-26.

[16] Fitzpatrick, K. \& LaGory, M. (2000). Unhealthy places - The ecology of risk in the urban landscape. New York and London: Routledge.

[17] Kohlhuber, M., Mielck, A., Weiland, S. K. \& Bolte, G. (2006). Social inequality in perceived environmental exposures in relation to housing conditions in Germany. Environ Res., 101, 246-255.
[18] Jakubowsky, M. (2011). Low level of environmental lead exposure and intellectual impairment in children - The current concepts of risk assessment. Int J Occup Med Envir Health, 24, 1-7.

[19] Department of Health and Social Security (1980). Inequalities in Health: Report of a Working Group. London: MHSO.

[20] Gallo, L. C., Smith, T. W., \& Cox, C. M. (2006). Socioeconomic status, psychosocial processes, and perceived health. An interpersonal perspective. Ann Behav Med., 31, 109-119.

[21] Kopp, M., Skrabski, Á., Székely, A., Stauder, A. \& William, R. (2007). Chronic stress and social changes: socioeconomic determination of chronic stress. Ann NY Acad Sci., 1113, 325-338.

[22] KangsenScammell, M., Senier, L., Darrah-Okike, J., Brown, P. \& Santos, S. (2009). Tangible evidence, trust and power: Public perceptions of community environmental health studies. Soc Sci Med., 68, 143-153.

[23] Varga, C. (2011). A history-based environmental health: On the frontiers of ecology, public health and history. WebmedCentral ECOLOGY 2(3):1-6. (http://www.webmedc entral.com/wmcpdf/Article_WMC001701.pdf)

[24] Varga, C., Kiss, I. \& Ember, I. (2002). The lack of environmental justice in Central and Eastern Europe. Environ Health Perspect., 110, A662-663.

[25] Sacks, J. D., Stanek, L. W., Luben, T. J., Johns, D. O., Buckeley, B. J., Brown, J.S., et al. (2011). Particle matter-induced health effects: Who is susceptible? Environ Health Perspect., 119, 446-454.

[26] Flores-Obando, R. E., Gollin, S. M. \& Ragin, C. C. (2010). Polymorphisms in DNA damage response genes and head and neck cancer risk. Biomarkers, 15, 379-399.

[27] Else, J. G. \& Pokras, M. A. (2002). Ecological Health and Change. Introduction. In: Aguirre et al. eds.: Conservation Medicine, Oxford University Press, New York, pp. 3-7. 